Article

\title{
Comparison of Metabolome and Transcriptome of Flavonoid Biosynthesis Pathway in a Purple-Leaf Tea Germplasm Jinmingzao and a Green-Leaf Tea Germplasm Huangdan reveals Their Relationship with Genetic Mechanisms of Color Formation
}

\author{
Xuejin Chen, Pengjie Wang, Yucheng Zheng, Mengya Gu, Xinying Lin, Shuyan Wang, Shan Jin * \\ and Naixing Ye* \\ College of Horticulture, Fujian Agriculture and Forestry University/Key Laboratory of Tea Science in University \\ of Fujian Province, Fuzhou 350002, China; 1180311002@fafu.edu.cn (X.C.); 2180311002@fafu.edu.cn (P.W.); \\ 1170311017@fafu.edu.cn (Y.Z.); 1190311005@fafu.edu.cn (M.G.); 1190311011@fafu.edu.cn (X.L.); \\ 1100311012@fafu.edu.cn (S.W.) \\ * Correspondence: 000q812072@fafu.edu.cn (S.J.); 000q020063@fafu.edu.cn (N.Y.)
}

Received: 4 May 2020; Accepted: 7 June 2020; Published: 11 June 2020

check for updates

\begin{abstract}
Purple-leaf tea is a phenotype with unique color because of its high anthocyanin content. The special flavor of purple-leaf tea is highly different from that of green-leaf tea, and its main ingredient is also of economic value. To probe the genetic mechanism of the phenotypic characteristics of tea leaf color, we conducted widely targeted metabolic and transcriptomic profiling. The metabolites in the flavonoid biosynthetic pathway of purple- and green-leaf tea were compared, and results showed that phenolic compounds, including phenolic acids, flavonoids, and tannins, accumulated in purple-leaf tea. The high expression of genes related to flavonoid biosynthesis (e.g., PAL and $L A R$ ) exhibits the specific expression of biosynthesis and the accumulation of these metabolites. Our result also shows that two CsUFGTs were positively related to the accumulation of anthocyanin. Moreover, genes encoding transcription factors that regulate flavonoids were identified by coexpression analysis. These results may help to identify the metabolic factors that influence leaf color differentiation and provide reference for future research on leaf color biology and the genetic improvement of tea.
\end{abstract}

Keywords: Camellia sinensis; leaf coloration; flavonoid biosynthesis; widely targeted metabolomic; transcriptomic; coexpression analysis

\section{Introduction}

Tea (Camellia sinensis (L.) O. Kuntze) is a perennial evergreen woody plant species that belongs to the Theaceae family of angiosperms [1]. Tea plants are grown widely in the tropical and subtropical zones around the world, including mainly China, Japan, India, and Kenya [2]. Purple-leaf tea, a novel germplasm of $C$. sinensis, has a unique color and a high content of bioactive flavonoids [3-5]. Flavonoids, as the most pronounced secondary metabolites in plants, play an important role in human health benefits and have potential physiological functions with antioxidant, antiaging, anticancer abilities and balance blood glucose. The intake of flavonoid composition in tea can reduce incidence of cardiovascular disease, cancer, diabetes, and hypertension [6,7].

Flavonoids, which belong to the phenolic class of compounds [8], include flavones, flavonoids, isoflavones, anthocyanins, flavanols, flavonols, and derivatives (e.g., catechins) $[9,10]$. Although flavonoids (including glycosyl derivatives) show a low threshold, they not only enhance the bitter effect of caffeine but also contribute to the astringency and bitterness of tea infusions [11]. 
Most genes have been actively studied for their contributions to flavonoid accumulation in many plants [12], including Arabidopsis (Arabidopsis thaliana) [13], maize (Zea mays) [14], figs (Ficus carica L.) [15], and tea plant (Camellia sinensis) [16]. The red color of cotton is mainly caused by flavonoid accumulation. Except for $4 C L$ and $F 3^{\prime} 5^{\prime} H$ of flavonoid-related genes in red cotton, other genes that regulate flavonoid biosynthesis have higher transcription levels than those of white cotton [17]. The enzymatic properties related to flavonoid accumulation in vitro have been confirmed $[18,19]$. Under the regulation of the CsFLS gene, the content of flavonoids in the tea of the albino cultivar "Rougui" is higher than that of normal green-leaf cultivars [20]. However, the regulatory pattern of flavonoid biosynthesis in purple-leaf tea not only differs from that of other currently ornamental plants but also does not match those characterized for regulating flavonoid in C. sinensis [21].

In addition to the synthetic enzymes involved in the pathway, regulation by TFs has been elucidated. Several TFs that regulate the expression of the structural flavonoid genes have been identified in tea plants. For example, MYB11, MYB12, and MYB111 play important roles in the biosynthesis of flavonol through the activation of upstream genes, such as $\mathrm{CHS}, \mathrm{CHI}$, and $\mathrm{FH}$, while the MYB-bHLH-WD40 (MBW) complex affects downstream genes that contribute to various branches, such as anthocyanins and procyanidin [22,23]. In tea plants, CsMYB2 promotes the expression of $C_{S F}{ }^{\prime} H$, whereas CsMYB26 is positively correlated with CsLAR expression [24]. MYB and $b H L H$ exert regulatory effects on EGCG biosynthetic genes in plants [19]. However, the molecular mechanism of the transcription regulation of flavonoid biosynthesis in purple-leaf tea remains unknown, and the gene regulatory network of flavonoid biosynthesis has not been fully investigated.

Here, we utilized an integrated widely targeted metabolomic and transcriptomic approach to reveal flavonoid biosynthesis in purple- and green-leaf tea. Our results provide new insights into the 49 flavonoid compounds that were accumulated, including six anthocyanins from purple-leaf tea. Additionally, we characterized several differentially expressed genes (DEGs) that were significantly up- or downregulated in flavonoid biosynthetic structural genes and transcriptional regulatory genes of flavonoid biosynthesis. Some of these genes were further verified by qRT-PCR. These findings revealed that the analysis of metabolic expression profiles and molecular regulation levels enriched the understanding of purple-leaf tea and provided valuable theoretical support for further exploration of new health products of purple-leaf tea.

\section{Results}

\subsection{Comparison of Morphological Phenotypes between JMZ and HD}

Significant differences between JMZ and HD were observed in the morphological phenotypes, especially concerning leaf color (Figure 1). The leaf of the cultivar JMZ exhibits lustrous surfaces and sharply jagged edges compared with HD. Furthermore, compared with HD, the color of buds and leaves on the young shoots was different from that of the old leaves in JMZ plant. The buds and young leaves were purple, but the old leaves on the lower part of the tea plant were green. 




Figure 1. The morphological phenotypes of the two tea cultivars. (A) Young shoot of C. sinensis cv. Jinmingzao (JMZ). (B) Purple leaves of JMZ plant. (C) Young shoot of C. sinensis cv. Huangdan (HD). (D) Green leaves of HD plant.

\subsection{Metabolite Difference between JMZ and HD}

To compare the metabolite difference of purple-leaf tea JMZ and green-leaf tea HD, dataset obtained from LC-ESI-MS/MS were subjected to PCA and PLS-DA analyses. The results revealed that purple-leaf tea JMZ and green-leaf tea HD clearly separated in score plots (Figure 2a,b). Furthermore, volcano plots were generated to display the significant differences between JMZ and HD (Figure 2c). In the present work, 435 metabolites were identified in purple-leaf tea JMZ and green-leaf tea HD. Among the metabolites, 127 differentially expressed metabolites (SCMs) were detected between purple-leaf tea $\mathrm{JMZ}$ and green-leaf tea HD, including 57 downregulated and 70 upregulated metabolites, respectively (Figure 2d). Many flavonoids and anthocyanins were upregulated in the purple-leaf tea JMZ. Moreover, some metabolites, including phenolic acids, amino acids, and derivatives, were downregulated.

To further compare the fold changes in SCM levels of purple-leaf tea JMZ and green-leaf tea HD, we generated a heat map of the 127 SCMs by using TBtools (Figure 3 and Supplementary Table S2), marked with green to represent elevated levels of metabolites and red to represent decrease. Forty-nine flavonoids were found to be differentially accumulated in purple-leaf tea JMZ and green-leaf tea HD, of which in purple-leaf tea JMZ, 37 flavonoids, including cyanidin 3-O-glucoside, delphinidin 3-O-glucoside, peonidin 3-O-glucoside chloride, cyanidin 3-rutinoside, cyanidin 3-O-galactoside, sissotrin, and 2'-hydroxygenistein, were significantly increased, whereas 12 flavonoids, including syringaldehyde, chrysoberyl $O$-glucuronic acid, 6-hydroxykaempferol-7,6-O-diglucoside, and quercetin 3,7-bis-O- $\beta$-D-glucoside, were decreased. Compared with green-leaf tea HD, 22 of the 26 significantly changed phenolic acids, including isochlorogenic acid A, 3,4-dicaffeoylquinic acid, neochlorogenic acid, isochlorogenic acid C, cryptochlorogenic acid, and chlorogenic acid methyl ester, were decreased in JMZ, whereas cynarin, cinnamic acid, and 2-methoxybenzoic acid were increased. All significantly changed 
amino acids and derivatives showed markedly increased abundance in purple-leaf tea JMZ, especially with a 3.34-fold increase. Seven out of the 10 significantly changed nucleotides and derivatives, including allopurinol, xanthosine, xanthine, inosine 5'-monophosphate, and 3-methylxanthine, showed markedly higher abundances in purple-leaf tea JMZ than in green-leaf tea HD. Among the six significantly changed alkaloids, four compounds, namely, fer-agmatine, N-feruloyl agmatine, indole, and isoquinoline, showed markedly higher abundances in purple-leaf tea JMZ than in green-leaf tea HD. Interestingly, four significantly changed organic acids were markedly decreased in green-leaf tea HD.
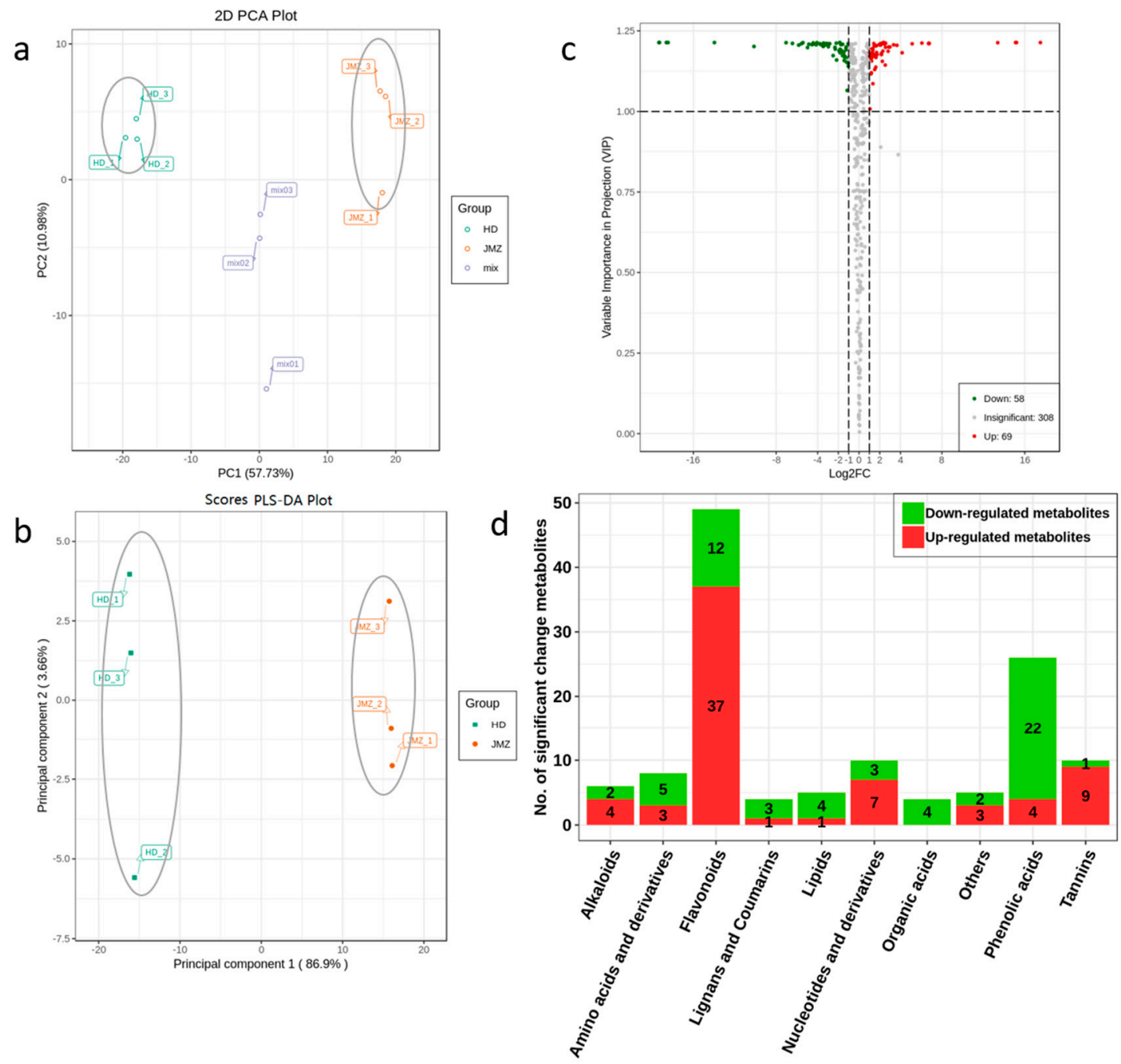

Figure 2. Multivariate statistical analysis of metabolites from C. sinensis cv. Jinmingzao (JMZ) and C. sinensis cv. Huangdan (HD). (a) PCA score plot of metabolites of the young shoots of JMZ (red) and $\mathrm{HD}$ (green); the $x$-axis represents the first principal component and the $y$-axis represents the second principal component. (b) PLS-DA score plot of metabolites between the young shoots of JMZ (red) and HD (green). (c) Volcano plots of metabolites between the young shoots of JMZ and HD. Metabolites with $q$-value $>0.05$ are highlighted in red for upregulation and in green for downregulation; and (d) The 127 SCMs were divided into 10 categories. 


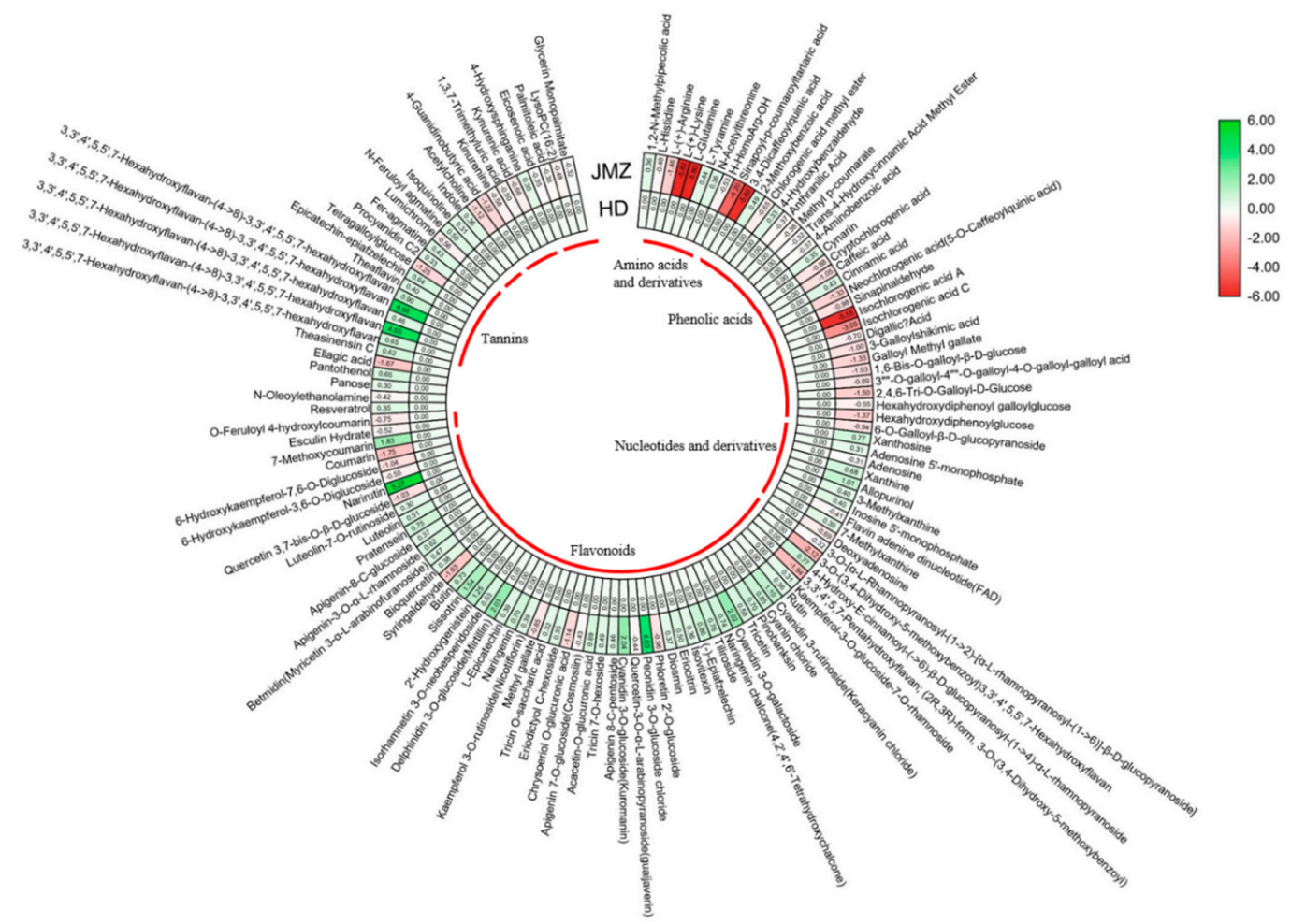

Figure 3. Heatmap of the fold change of significantly changed metabolites (SCMs) between the young leaves of C. sinensis cv. Jinmingzao (JMZ) and C. sinensis cv. Huangdan (HD). The color bar represents the normalized fold change values. Five categories with elevated SCM levels, including amino acids and derivatives, phenolic acids, nucleotides and derivatives, flavonoids, and tannins, are marked.

\subsection{Accumulation Patterns of Phenolic Acids and Tannins in JMZ and HD}

Phenolic acids are aromatic secondary metabolites that are widely distributed in the plant kingdom [25]. Some phenolic acids such as gallic acid have attracted considerable attention because of their powerful antiradical, antioxidative, antitumor, and antimicrobial properties [26-28]. The heatmap identified 26 significantly different metabolites in leaves of the two different cultivars (Figure 3). The contents of most of the significant metabolites, except for chlorogenic acid methyl ester, cynarin, cinnamic acid, and 2-methoxybenzoic acid, were lower in purple-leaf tea JMZ than in green-leaf tea HD. Therefore, the phenolic acid contents may be negatively correlated with purple-leaf tea and positively correlated with green-leaf tea.

Tannins are important compounds that enhance the color stability and the anthocyanin content [29]. Ten tannins, namely, theasinensin C (T1), 3,3', 4', 5, 5' ,7-hexahydroxyflavan-(4->8)-3,3', $4^{\prime}, 5,5^{\prime}, 7-$ hexahydroxyflavan (T2), 3,3', 4', 5, 5',7-hexahydroxyflavan-(4->8)-3,3' , 4', 5, 5' ,7-hexahydroxyflavan (T3), 3, $3^{\prime}, 4^{\prime}, 5,5^{\prime}, 7$-hexahydroxyflavan-(4->8)-3,3', $4^{\prime}, 5,5^{\prime}, 7$-hexahydroxyflavan $\quad$ (T4), 3, $\quad 33^{\prime}, 4^{\prime}, 5,5^{\prime}, 7-$ hexahydroxyflavan-(4->8)-3,3' $4^{\prime}, 5,5^{\prime}, 7$-hexahydroxyflavan (T5), 3, $3^{\prime}, 4^{\prime}, 5,5^{\prime}, 7$-hexahydroxyflavan(4->8)-3,3', 4',5,5',7-hexahydroxyflavan (T6), theaflavin (T7), epicatechin-epiafzelechin (T8), tetragalloylglucose (T9), and procyanidin C2 (T10), showed significant discrepancies between purple-leaf tea JMZ and green-leaf tea (Figure 3). Nine of these tannins were significantly increased in purple-leaf tea. T3 and T5 showed 15.203- and 15.114-fold changes, respectively, in purple-leaf tea compared with green-leaf tea. However, only one metabolite in JMZ, namely, tetragalloylglucose (T9), was upaccumulated in tannin biosynthesis.

\subsection{Differential Expression of Genes in JMZ and HD}

To obtain a reference transcriptome for the purple-leaf and green-leaf tea, we constructed an RNA-seq library with the total RNA of tea leaf samples. A total of 37.98-49.55 million raw reads 
were obtained by using the Illumina Hi-Seq 4000 platform. After deleting reads containing adapters or poly-N and low-quality reads, high-quality data with a Q30 percentage of $90.03 \%-90.58 \%$ and GC percentage of $45.10 \%-45.58 \%$ were available for analysis (Table 1 and Supplementary Table S3). All the unigenes were successfully annotated through alignment to the reference database with 35,967,226 (93.18\%), 34,512,682 (93.41\%), 41,291,024 (93.06\%), 44,416,160 (93.33\%), 45,021,912 (93.64\%), and $41,274,294(93.99 \%)$. All transcriptome data sets were stored in the NCBI SRA database under the accession number PRJNA638184.

Table 1. Quality of the transcriptomes of young shoots between C. sinensis cv. Jinmingzao (JMZ) and C. sinensis cv. Huangdan (HD).

\begin{tabular}{cccccc}
\hline Sample & Raw Reads & Clean Reads & Q30 (\%) & GC (\%) & Mapped Reads \\
\hline JMZ_1 & 39848584 & 38598850 & 90.48 & 45.51 & $35967226(93.18 \%)$ \\
JMZ_2 & 37983438 & 36946120 & 90.23 & 45.58 & $34512682(93.41 \%)$ \\
JMZ_3 & 45639484 & 44371332 & 90.38 & 45.50 & $41291024(93.06 \%)$ \\
HD_1 & 49081330 & 47588286 & 90.05 & 45.24 & $44416160(93.33 \%)$ \\
HD_2 & 49552984 & 48077760 & 90.58 & 45.10 & $45021912(93.64 \%)$ \\
HD_3 & 45187036 & 43911796 & 90.03 & 45.31 & $41274294(93.99 \%)$ \\
\hline
\end{tabular}

We annotated the GO and KEGG pathway functional enrichment analyses by using the Blast-GO and KEGG pathway databases, respectively. The DEGs were divided into three main categories, namely, molecular function, biological process, and cellular component, and 49 subcategories of the GO classification. In the group, 859, 648, and 213 unigenes were assigned to the biological process, molecular function, and cellular component terms. These genes were further classified into 48 functional subcategories based on mapped homology (Figure 4A). Genes in the biological process category were primarily matched and classified into metabolic processes, cellular process, and single-organism processes. In the molecular function term, most of the unigenes exhibited catalytic activity, binding, and transporter activity. The most abundant GO terms in the cellular component category included the membrane, cell, cell part, organelle, and organelle part. In the KEGG pathway-enrichment analysis, matches were found for 3916 unigenes, which were mapped to 116 KEGG pathways. According to the KEGG pathway database, the main enriched metabolic process was phenylpropanoid biosynthesis in JMZ and HD (Figure 4B).

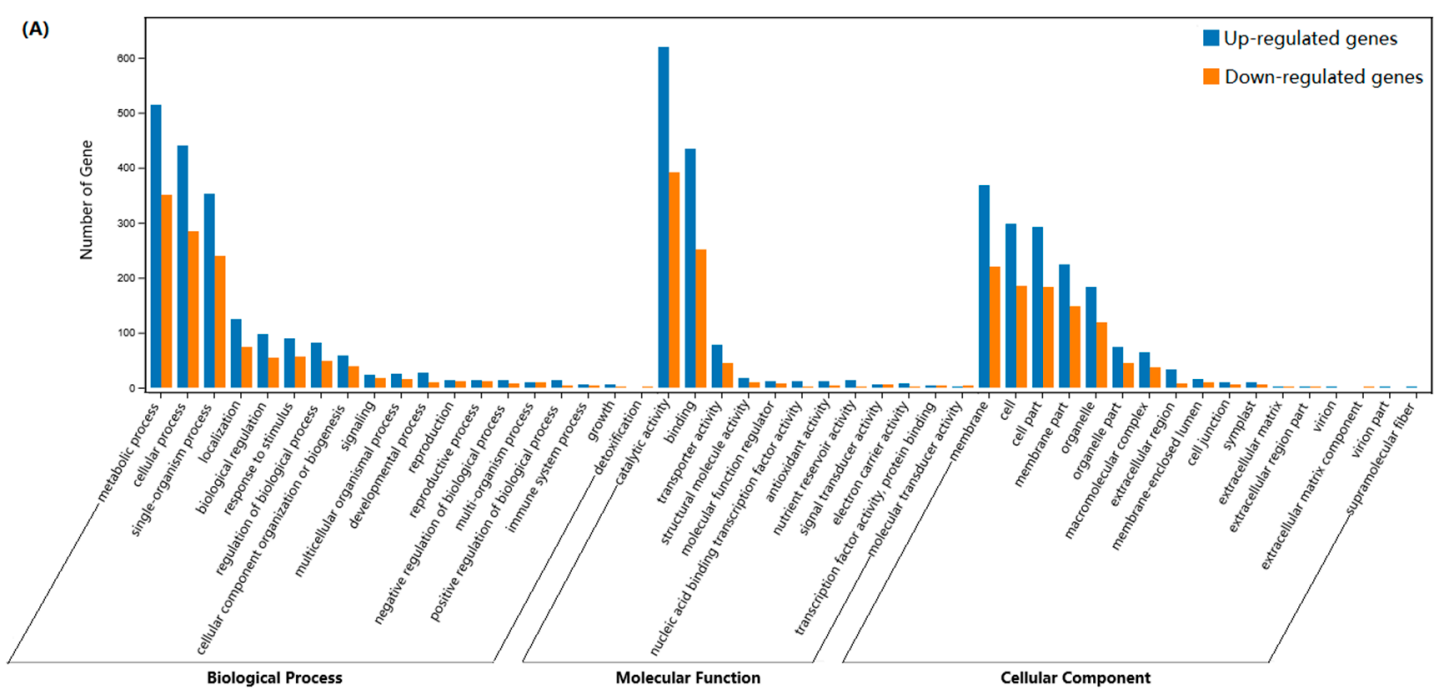

Figure 4. Cont. 


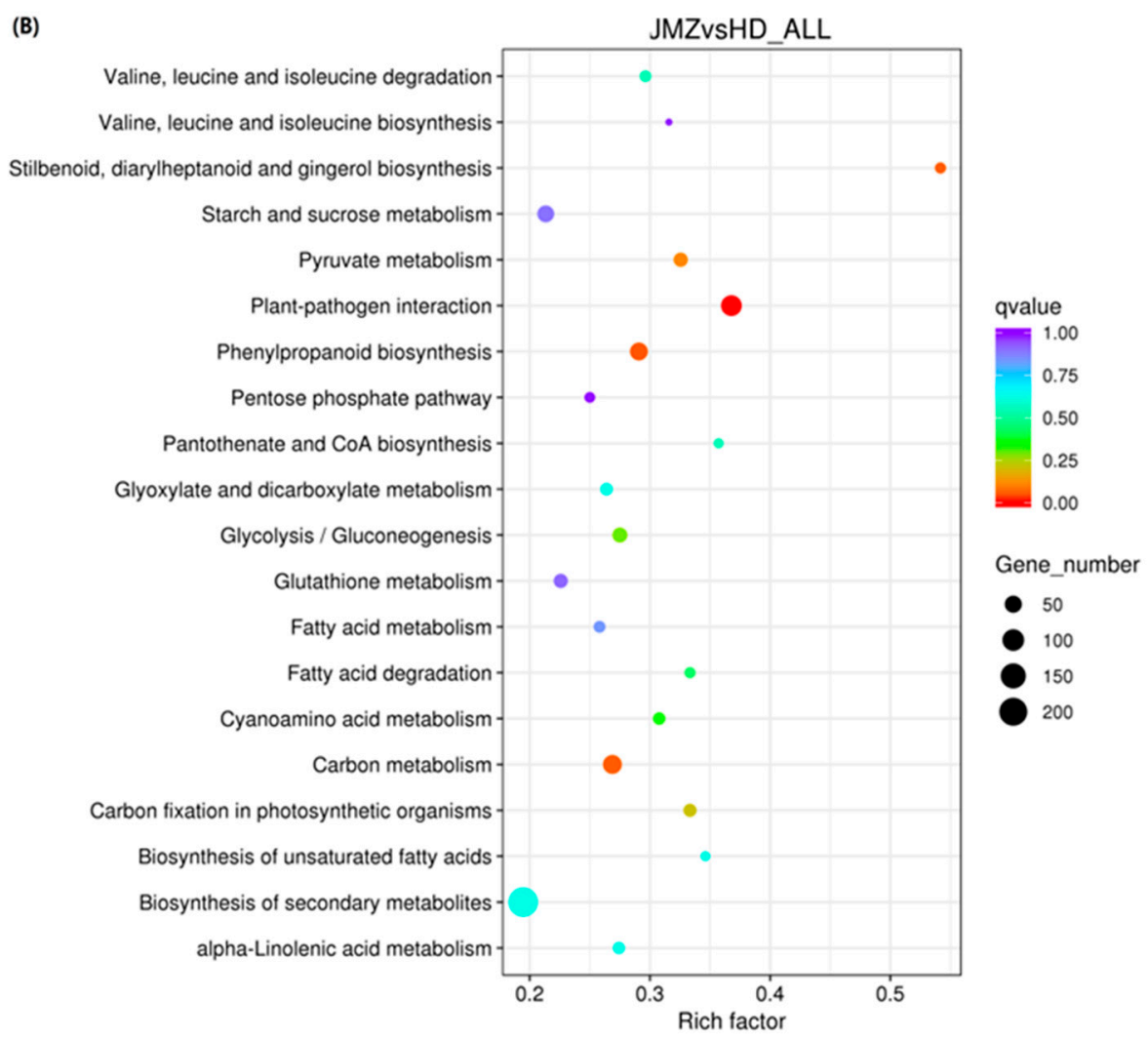

Figure 4. GO classification and KEGG pathway enrichment of differentially expressed genes in JMZ and HD. (A) The GO terms were classified into three categories: molecular function, cellular component, and biological process categories. The top 48 enriched GO terms were exhibited in each cluster. (B) Top 20 enriched KEGG pathway enrichment of differentially expressed genes (DEGs) of tea cultivar between JMZ and HD. The significantly changed pathways (Q-value $<0.05$ ) are related to phenylpropanoid biosynthesis.

\subsection{Analysis of DEGs and SCMs Related to the Flavonoid Biosynthesis Pathway in JMZ and HD}

Based on the flavonoid metabolite results, the pattern of differentially accumulated flavonoids was significant when the ratio $>2$ or ratio $\leq 1 / 2$, q-value $\leq 0.05$, and VIP $\geq 1$ between purple- and green-leaf tea. Among the peaks that indicated differentially accumulated metabolites, 49 were attributed to flavonoids.

Flavonoids are synthesized via the phenylpropanoid pathway (Figure 5) through the transformation of phenylalanine into 4-coumarol-CoA, which ultimately enters the flavonoid biosynthesis pathway. Enrichment analysis revealed that 17 genes were involved in the flavonoid biosynthesis pathway in purple-leaf tea JMZ [30]. However, we only detected PAL, C4H, 4CL, DFR, LAR, ANS, and UFGT, whereas CHS, CHI, F3H, F3' $5^{\prime} H$, and $F 3^{\prime} H$ were not detected. We observed phenylalanine, cinnamic acid, p-coumaric acid, delphinidin-3-glucoside, cyanidin 3-rutinoside, cyanidin 3-O-galactoside, and cyanidin 3-O-glucoside by widely targeted metabolomics. Significant differences were observed in the expression of enzymes detected between the two different colored tea cultivars. Interestingly, the gene downstream in the flavonoid biosynthesis pathway differed most significantly among the different tea cultivars. For example, in purple-leaf tea JMZ, the principal DEGs were TEA024758, TEA023829, and TEA015762, which encode DFR and ANS. Genes involved in LAR and UFGT presented high expression levels in purple-leaf tea JMZ. The final step in the anthocyanin biosynthesis pathway is 3-glucoside formation by uridine $5^{\prime}$-diphosphate (UDP)-glucose: UFGT. The expression of the UFGT gene was active in the last step of anthocyanin 
modifications. The existence of anthocyanins and the generation of purple pigmentation in cells were stable because of the role of the abovementioned genes [31].

(A)

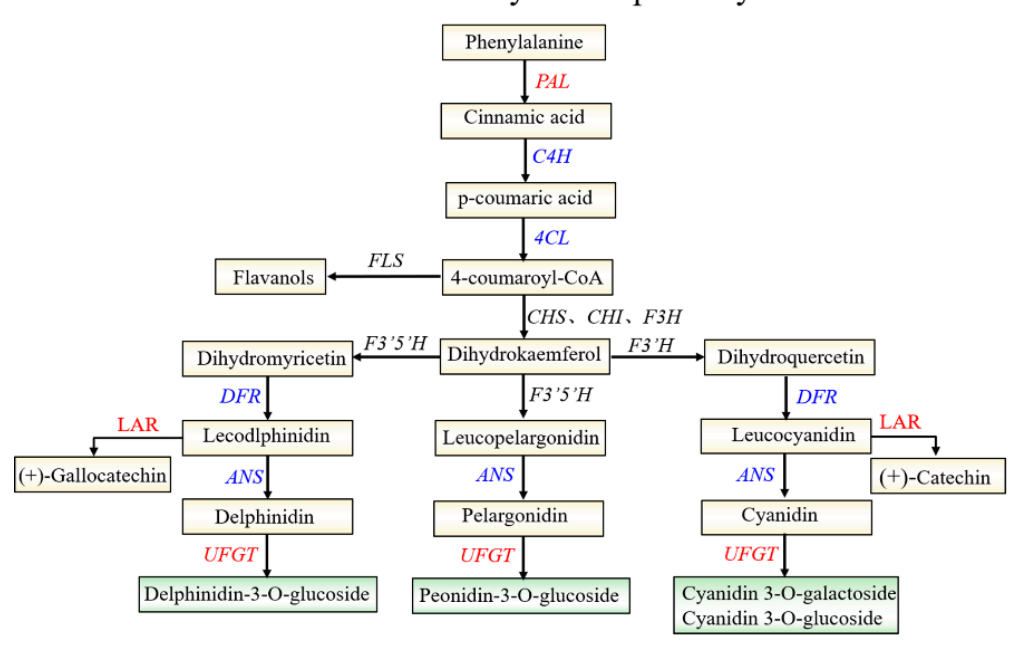

(B)

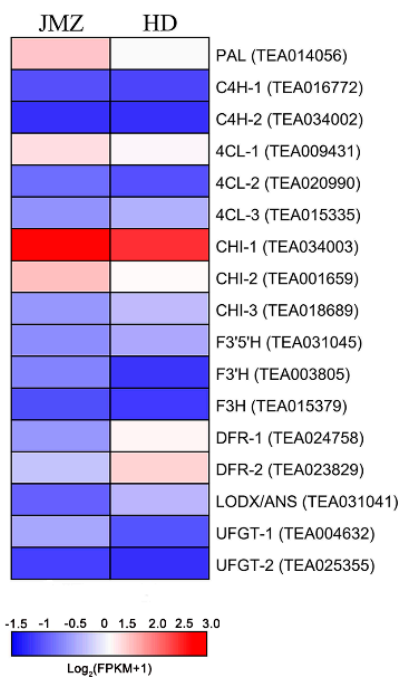

Figure 5. Transcript profiling of genes in the phenylpropanoid and flavonoid biosynthetic pathways in C. sinensis cv. Jinmingzao (JMZ) and C. sinensis cv. Huangdan (HD). (A) Flavonoid biosynthetic pathway. Proposed pathway of biosynthesis derived from the literature [30]. PAL, phenylalanine ammonia-lyase; $\mathrm{C} 4 \mathrm{H}$, cinnamic acid 4-hydroxylase; $4 \mathrm{CL}$, 4-coumarate $\mathrm{CoA}$ ligase; $\mathrm{CHS}$, chalcone synthase; $C H I$, chalcone isomerase; $F 3 H$, flavanone 3-hydroxylase; $F 3^{\prime} H$, flavonoid $3^{\prime}$-hydroxylase; $D F R$, dihydroflavonol flavonol synthesis; ANS/LDOX, anthocyanidin synthase/leucocyanidin oxygenase; LAR, leucocyanidin reductase; UFGT, UDP glucose-flavonoid 3-o-glcosyl-transferase. (B) Heat map representation of the expression patterns of flavonoid-related genes. Gene expression is displayed as heat map depicting the log2 (FPKM) values. Red and blue font indicate up- and downregulated genes, respectively

\subsection{Confirmation of Flavonoid Regulatory Genes by Using qRT-PCR}

To further validate our RNA-seq expression profile data, we performed qRT-PCR assays on 17 selected structural genes, including one $P A L$, two $C 4 H$, three $4 C L$, three $C H I$, one $F 3^{\prime} 5^{\prime} H$, one $\mathrm{F}^{\prime} \mathrm{H}$, one $F 3 H$, two DRF, one LODX/ANS, and two UFGT. With respect to the purple-leaf tea, $6 / 17$ (35\%) were found to be inconsistent with respect to expression between the qRT-PCR and RNA-Seq data. Overall, 11/17 (65\%) were consistent with respect to expression between the qRT-PCR and RNA-Seq data. Those included CsPAL, CsC4H-1, Cs4CL, Cs4CL-2, CsCHI-1, CsCHI-2, CsF3' $5^{\prime} H, C_{s} F 3^{\prime} H, C s D F R-1$, CsUFGT-1, and CsUFGT-2. Detailed information of the main flavonoids-related up- and downregulated genes is presented in Figure 6. 

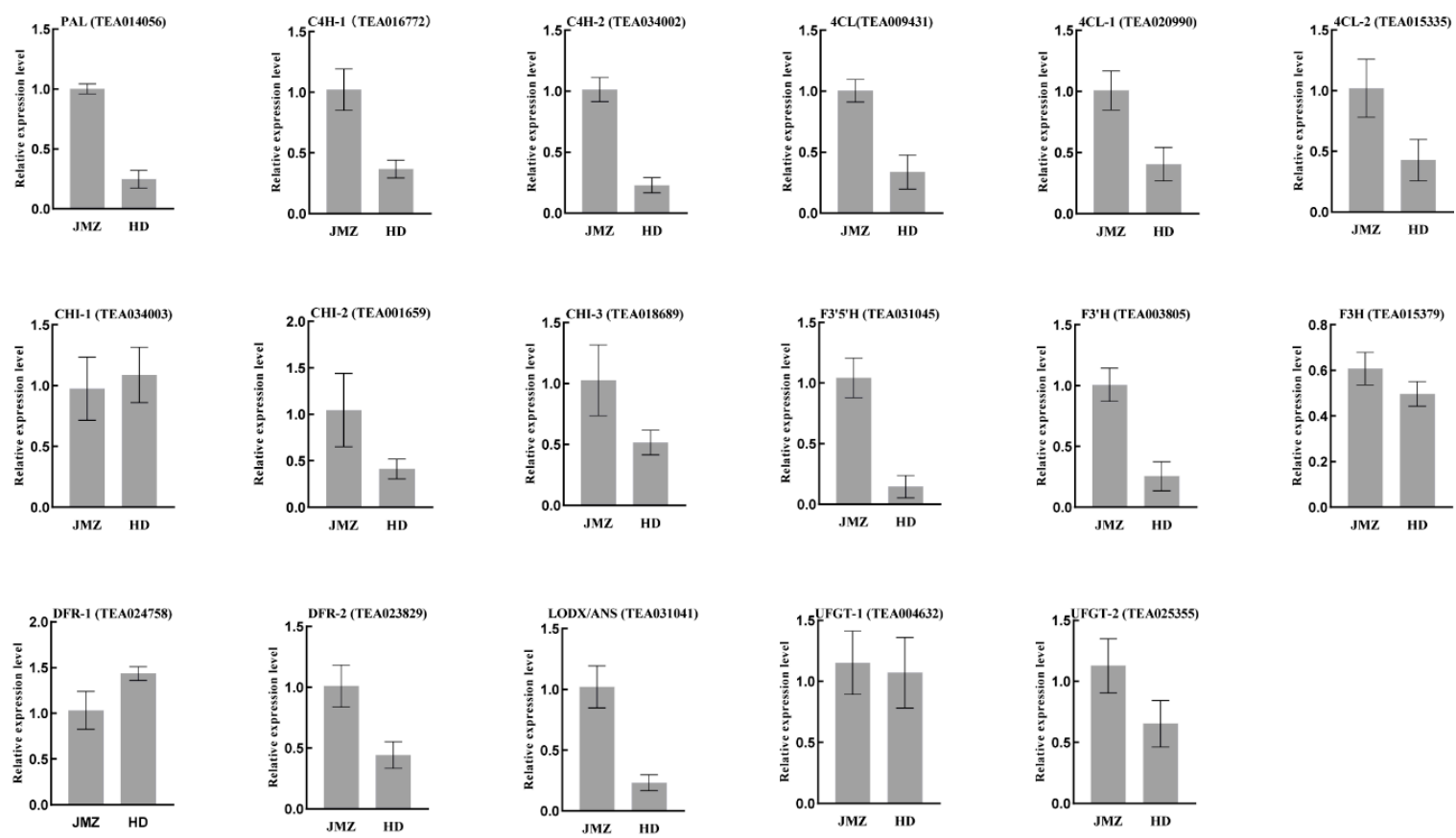

Figure 6. Expression of 17 selected candidate genes in purple-leaf and green-leaf tea by qRT-PCR validation. $P A L$, phenylalanine ammonia-lyase; $C 4 H$, cinnamic acid hydroxylase; $4 C L$, coumadin CoA ligase; $C H S$, chalcone synthase; $C H I$, chalcone isomerase; $F 3 H$, flavonoid 3-hydroxylase; $F 3^{\prime} H$, flavonoid $3^{\prime}$-hydroxylase; $F 3^{\prime} 5^{\prime} H$, flavonoid $3^{\prime} 5^{\prime}$-hydroxylase; $D R F$, dihydroflavonol 4-reductase; $L A R$, leucoanthocyanidin reductase; UFGT, UDP glucose-flavonoid 3-o-glcosyl-transferase, and SCPL1A, type $1 \mathrm{~A}$ serine carboxypeptidase-like acyltransferases. The $x$-axis represents two different tea cultivars of "JMZ" and "HD," and the $y$-axis represents relative expression. The data represent the mean from three replicates with three biological repeats. Error bars indicate SE.

\subsection{Conjoint Analysis between Transcripts and Flavonoid Derivative}

The inferred networks were confirmed by Pearson correlation analysis based on the data from metabolism, transcriptome, and transcription factors in the flavonoid biosynthesis of purple-leaf tea JMZ. Eleven structural genes in the phenylpropanoid-flavonoid pathways, including $P A L, C 4 H, 4 C L$, $D F R, L A R, A N S$, and UFGT, were the main regulators. We further analyzed the transcription factor families involving MYB, bHLH, and WD40, which perform crucial roles in flavonoid biosynthetic structural genes; among the detected transcription factors, 66 MYBs and $187 \mathrm{bHLH}$ were identified. Structural genes in the phenylpropanoid-flavonoid pathways and two TFs ( $M Y B$ and $b H L H$ ) were used to coexpress the network and identify the genes that may regulate flavonoid compounds (Figure 7). These results suggested that the flavonoid DEGs and TFs may contribute to flavonoid biosynthesis in DEMs.

To further understand the regulatory pattern of the structural genes involved in the flavonoid biosynthesis pathways by transcripts, we performed gene coexpression analysis. The correlations between the DEGs in the flavonoid pathway illustrated the metabolites in the pathway (Figure 7A). According to our dataset, the networks revealed the presence of a hub gene, namely, "shikimate O-hydroxycinnamoyltrasferase" (CsHCT, TEA029054), in purple-leaf tea JMZ. The expression level of CsHCT was identified that was positively related to the contents of flavonoids, dihydroflavone, isoflavones, chalcones, anthocyanins, flavanols, dihydroflavonol, and flavonoid carbonoside in purple-leaf tea JMZ. Therefore, the results indicated that CsHCT is a key regulator of the flavonoids biosynthesis in purple-leaf tea JMZ. Furthermore, we also obtained the transcription factor that may be involved in the regulation of flavonoid biosynthesis. Correlation calculations between these identified differentially expressed $M Y B$ and $b H L H$ TFs and DEMs were conducted via coexpression analysis. 
The results showed that 45 MYB TF expressed a high correlation with four flavonoids (flavonoid carbonoside, flavanols, dihydroflavonol, and anthocyanins), whereas 51 bHLH TF expressed a high correlation with nine flavonoids (flavonoids, dihydroflavone, isoflavones, chalcones, anthocyanins, flavanols, flavonols, dihydroflavonol, and flavonoid carbonoside) $(r>0.9$, Figure 7B,C). Notably, zinc finger A20 (TEA016255), GPI-anchored protein (TEA032923), and F-box/LRR-repeat protein (TEA000314), which are group members of the R2R3-MYB TFs, were the key dominant factors of flavonoid biosynthesis and closely positively related to the four flavonoids (anthocyanins, flavanols, dihydroflavonol, and flavonoid carbonoside). The genes, namely, bHLH121 (TEA019255), MRS2-F (TEA008908), actin-depolymerizing factor (TEA020752), and MOS14 (TEA025704) had the highest degree of connectivity with flavonoid synthesis metabolites. Thus, CsHTS, MYB, and $b H L H$ were identified as key genes for flavonoid synthesis.

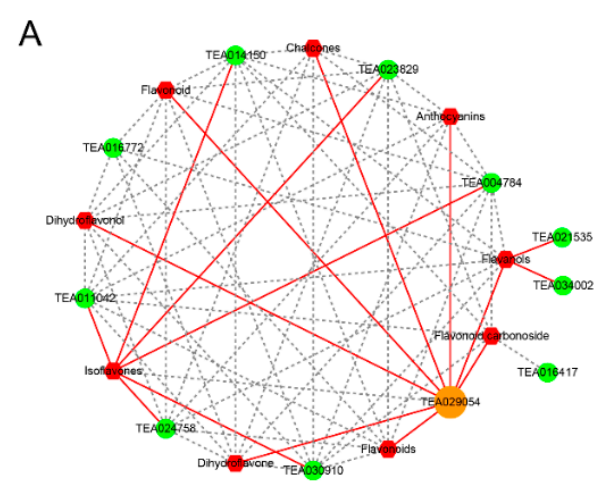

B
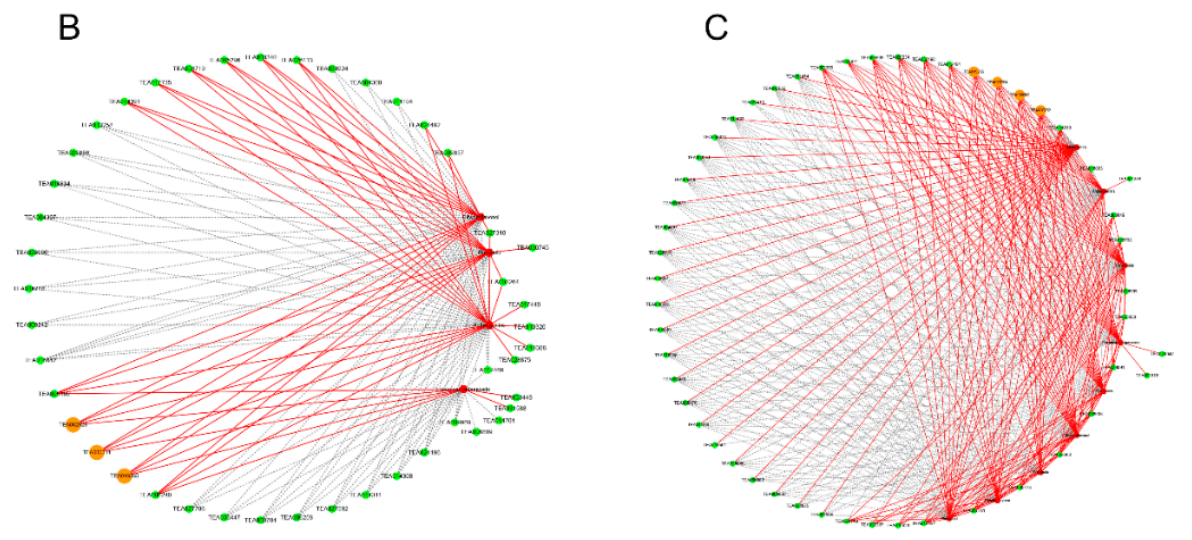

Figure 7. Connection network between flavonoid biosynthesis-related DEGs and differential accumulated flavonoid metabolites in purple-leaf tea JMZ. (A) Connection network between flavonoid biosynthesis structural genes and flavonoid metabolites, (B) connection network between MYB transcription factor genes and flavonoid metabolites, and (C) connection network between $b H L H$ transcription factor genes and flavonoid metabolites. The green circle represents the key regulator of flavone and flavonol biosynthesis.

\subsection{Catechin Content in JMZ and HD}

Catechins are responsible for astringency and bitterness [8]. A schematic diagram of catechin biosynthesis is shown in Figure 8. The content of C, EC, EGCG, EGC, GC, and ECG in JIM and HD were compared (Table 2). The EC, EGCG, EGC, GC, and ECG content were significantly higher in purple-leaf tea (JMZ) than those in the control leaves (HD). The results showed that the EC, EGCG, and ECG were predominant catechin compounds in JMZ, and their contents reached to 18.58, 55.17, and $22.88 \mathrm{mg} / \mathrm{g}$, respectively. The total catechin amounts in JMZ were significantly (1.4 folds) higher than those in HD. 


\section{Catechin biosynthetic pathway}

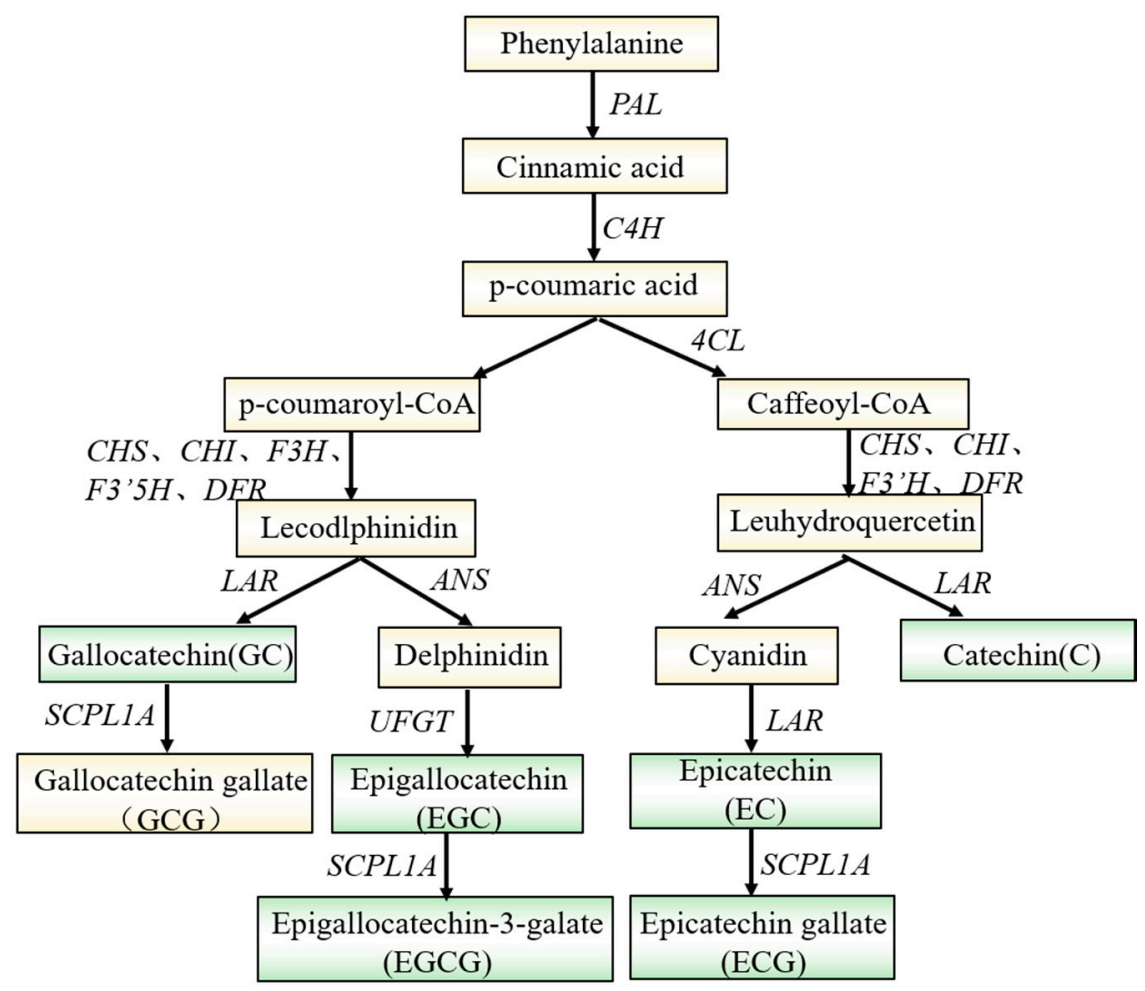

Figure 8. Catechin biosynthesis pathway. Proposed pathway of biosynthesis derived from the literature [32]. $P A L$, phenylalanine ammonia-lyase; $C 4 H$, cinnamic acid hydroxylase; $4 C L$, coumadin CoA ligase; $C H S$, chalcone synthase; $C H I$, chalcone isomerase; $F 3 H$, flavonoid 3-hydroxylase; $F 3^{\prime} H$, flavonoid $3^{\prime}$-hydroxylase; $F 3^{\prime} 5^{\prime} H$, flavonoid $3^{\prime} 5^{\prime}$-hydroxylase; $D R F$, dihydroflavonol 4-reductase; $L A R$, leucoanthocyanidin reductase; UFGT, UDP glucose-flavonoid 3-o-glcosyl-transferase, and SCPL1A, type 1 A serine carboxypeptidase-like acyltransferases.

Table 2. Content of catechins in JMZ and HD. All data are shown as the mean \pm SE. (* means significant difference, $p<0.05$ ).

\begin{tabular}{cccccccc}
\hline \multirow{2}{*}{$\begin{array}{c}\text { Tea } \\
\text { Cultivar }\end{array}$} & C & EC & EGCG & EGC & GC & ECG & Total Content \\
\cline { 2 - 7 }$(\mathbf{m g} / \mathbf{g})$
\end{tabular}

(+)-catechin (C), (-)-epicatechin (EC), (-)-epigallocatechin gallate (EGCG), (-)-epigallocatechin (EGC),

$(-)$-gallocatechin (GC), (-)-epicatechin gallate (ECG).

\section{Discussion}

The unique and delightful leaf color of tea is mediated by specific metabolic compounds and gene expression. The association of metabolite abundances and gene expression has been attracted increasing interest in recent years. In the present study, integrative analysis of metabolome and transcriptome profiles was performed to elucidate the mechanism of color formation in JMZ, a novel germplasm in China. The extensive metabolome datasets of JMZ were obtained for the first time in this study, which could provide a theoretical base for further study on mechanism of formation of pigments and genetic improvement of purple-leaf tea germplasm.

The complex network of metabolite profiling is remarkably changed in the diverse color of tea leaves [33]. The transcriptome and metabolome results of purple-leaf tea (JMZ) and green-leaf tea (HD) suggested that different molecular programs are involved in metabolites biosynthesis. In $C$. sinensis $\mathrm{cv}$. Zixin, high levels of bioactive compounds, e.g., flavonoid, chlorophyll, and carotenoid, are important 
for the formation of purple-leaf coloration [4]. In the current research, six anthocyanin components were detected in young shoots, including delphinidin 3-O-glucoside, cyanidin 3-O-glucoside, peonidin 3-O-glucoside chloride, cyanidin 3-O-galactoside, cyanidin 3-rutinoside, and cyanidin chloride. Among them, the contents of peonidin 3-O-glucoside chloride, cyanidin 3-O-glucoside, and delphinidin 3-O-glucoside in JMZ were significantly higher than those in HD, which were 13.39, 6.78, and 6.73 times higher, respectively. The main concern is composition of anthocyanin 3-O-glucoside in the cultivars of purple-leaf tea, that agreed with the previous reports of Zhou et al. [34] who observed that the anthocyanin 3-O-glucoside composition was mainly differentially accumulated at developmental stages in various colored tea flowers. Moreover, "Sunrouge," as one of the anthocyanin-rich teas in Japan, has identified four anthocyanins, including delphinidin-3-O- $\beta$-D-(6-(E)-p-coumaroyl)glucopyranoside, cyanidin-3-O- $\beta$-D-(6-(E)-p-coumaroyl)glucopyranoside, delphinidin-3-O- $\beta$-D-(6-(E)-pcoumaroyl) galactopyranoside, and cyanidin-3-O- $\beta$-D-(6-(E)-pcoumaroyl) galactopyranoside [35]. We also found other differentiation of phenolic compounds. In particular, many catechins, including EC, EGCG, EGC, GC, and ECG, were significantly increased in JMZ. The diversity and abundance of catechins are involved in the characteristic taste of some tea cultivars [36]. For instance, $70 \%-75 \%$ of the bitterness and astringency of green tea are related to these catechins [8]. Briefly, presence of most of the significantly changed flavonoids, anthocyanins, and catechins contents in the purple-leaf tea (JMZ) suggests that it may have high application value.

Previous studies have identified some key structural genes from the flavonoid pathways regulating coloration [37]. Our data showed six differentially expressed genes in JMZ, including $P A L, C 4 H, 4 C L$, $D F R, A N S$, and UFGT genes. PAL is as an important modulate pinpoint that regulates the flavonoid accumulation in the $C$. sinensis cv. Zijuan [38]. In agreement with other results, $P A L$ was proposed as a pivotal gene responsible for regulating the purple pigmentation in JMZ. Furthermore, $C 4 H$, $4 C L, D F R$, and ANS are the key branch-point genes that regulate the accumulation of flavonoid [39]. Our studies showed that low expression of $C 4 H, 4 C L, D F R$, and ANS may have a negative correlation to total anthocyanins in purple-leaf tea JMZ. Previous studies showed that $P A L, C 4 H$, and ANS genes were upregulated in the $C$. sinensis $\mathrm{cv}$. Foshou, which may contribute to the formation of anthocyanin compounds [40]. Besides, UFGT is the last enzyme in anthocyanin biosynthetic pathway, and it is the key to anthocyanin stability and water solubility [41]. The expression of UFGT genes were detected in red grape cultivars, but not in white cultivars [42]. A similar result was reported by Yonekura-Sakakibara et al. (2008) for Arabidopsis [43]. They found that flavonoid 3-O-glucosyltransferase (AtUDT78D2) catalyzes the glycosylation of both flavonol and anthocyanin, and the Arabidopsis UGT78D2 mutant exhibits a high anthocyanin level. Interestingly, we investigated the UFGT-like gene expressed in JMZ and isolated two genes, namely, CsUFGT1 (TEA004632) and CSUFGT2 (TEA004632), which are associated with anthocyanin accumulation in purple-leaf tea.

Our results confirmed that a novel gene, $H C T$, was characterized by interaction analysis of metabolism and different genes of flavonoid biosynthesis pathway. Although some HCT genes were studied, their function is still unclear. The expression of HCT displayed that the expression of HCT gene was repressed on the lignin biosynthesis of Arabidopsis [44], and HCT positively regulates flavone and flavonol biosynthesis in Euscaphis konishii Hayata [45]. In the present study, the expression of CsHCT positively related to the contents of flavonoids, dihydroflavone, isoflavones, chalcones, anthocyanins, flavanols, dihydroflavonol, and flavonoid carbonoside in purple-leaf tea JMZ. Therefore, these results demonstrated that HCT may be a key modulator to promote the flavonoid biosynthesis in plants. However, the relationship between HCT and the catalysis formation of flavonoids needs for further verification.

Furthermore, the abundant TFs with regulatory function in flavonoid biosynthesis belong to R2R3-MYB and bHLH protein family [46]. A positive or negative correlation exists between MYB TFs, which are the key regulators encoding structural genes involved in flavonoid biosynthesis $[47,48]$. In Tartary buckwheat (Fagopyrum tataricum), the AtMYB12 of MYB TFs members was the main regulator in the four flavanones [47]. The function and regulatory relationships between R2R3-MYB and the 
flavonoid biosynthesis have been discussed in crabapple [49]. In the present study, we identified three members of the subgroup R2R3-MYB TFs, namely, Zinc finger A20 (TEA016255), GPI-anchored protein (TEA032923), and F-box/LRR-repeat protein (TEA000314), which are closely related to four flavonoids, namely, anthocyanins, flavanols, dihydroflavonol, and flavonoid carbonoside. The regulation of flavonoid biosynthesis involves different bHLH TFs targeting genes [50,51]. In Arabidopsis, AtbHLH8 (PIF3) bound and activated the expression of anthocyanin synthesis-related genes, including $\mathrm{CHS}$, $F 3 H, D F R$, and ANS [52]. In the present study, we elucidated that the expression levels of $b H L H 121$ (TEA019255), MRS2-F (TEA008908), actin-depolymerizing factor (TEA020752), and MOS14 (TEA025704) were highly correlated with the contents of flavonoid synthesis metabolites.

\section{Materials and Methods}

\subsection{Tea Plant Materials}

Two four-year-old tea cultivars, a C. sinensis cv. Jinmingzao (JMZ) with purple-leaf and a C. sinensis cv. Huangdan (HD, a widely grown variety in China) with green-leaf tea, were cultivated on a tea plantation of Wuqu in Fuan city, Fujian Province, China (E119 $66^{\prime}$ N27 $10^{\prime} ; 150-200 \mathrm{~m}$ above sea level). For each cultivar, one bud and two young shoots were harvested from 24 individuals used for the metabolite analysis ( 3 biological replication $\times 4$ individual for each replicate) and RNA-seq ( 3 biological replication $\times 4$ individual for each replicate) on March 31, 2019. Samples were randomly collected from different branches of tea plants of each cultivar. All materials were frozen immediately in liquid nitrogen and then stored at $-80^{\circ} \mathrm{C}$ until further analysis. The DEGs and metabolites of purple-leaf and green-leaf tea were identified by transcriptome and widely targeted metabolomics, and functional genes with significant differences were verified by qRT-PCR. Six libraries, namely, JMZ_1, JMZ_2, JMZ_3, HD_1, HD_2, and HD_3 was constructed during the experiment.

\subsection{LC-ESI-MS/MS Analysis and Differential Metabolite Identification}

After freezing the tea samples with liquid nitrogen, a mixer-mill with zirconia ( $\mathrm{Zr} ; \mathrm{Z}=40$ ) beads (MM 400, Retach) was used to grind the samples at $30 \mathrm{~Hz}$ for $90 \mathrm{~s}$ until they were powdered. Precisely, $100 \mathrm{mg}$ of powdered sample was weighed and dissolved in $1.0 \mathrm{~mL}$ of $70 \%$ methanol extraction solution. The dissolved sample was stored overnight in a refrigerator at $4{ }^{\circ} \mathrm{C}$. Three vortices were used to improve the extraction rate. The mixture $(5 \mu \mathrm{L})$ was injected into an LC-ESI-MS/MS (LC, Shim-pack UFLC Shimadzu CBM30A system; ESI, MS, Applied Biosystems 6500 QTRAP ${ }^{\circledR}$ ) system. The detection was carried out as previously described [53]. Chromatographic separation was executed by an ACQUITY UPLC HSS T3 C18 $(1.8 \mu \mathrm{m}, 2.1 \mathrm{~mm} \times 100 \mathrm{~mm}$; Waters). The mobile phase consisted of A containing $0.04 \%$ acetic acid in deionized water and B containing acetonitrile with $0.04 \%$ acetic acid in acetonitrile. The A: B $(v / v)$ elution profile was as follows: 0-11 $\mathrm{min}, 95 \mathrm{~A}: 5 \mathrm{~B} ; 11-12 \mathrm{~min}, 5 \mathrm{~A}: 95 \mathrm{~B} ; 12.1-15 \mathrm{~min}$, and 95A:5B. The flow rate was maintained at $0.4 \mathrm{~mL} / \mathrm{min}$, and the column temperature was $40^{\circ} \mathrm{C}$.

The concentrations of metabolites were identified based on a widely targeted metabolomic method of Wuhan Maiteville Biotechnology Co., Ltd. (Wuhan, China). This method has been described in a study on tomatoes [54]. Metabolite identification was based on the parametric values $(\mathrm{m} / \mathrm{z}$ data, retention time, and fragmentation partners) and compared with the self-built database (MetaWare) for annotation results. Principal component analysis (PCA) and partial least-squares discriminant analysis (PLA-DA) were conducted to identify the significantly changed metabolites (SCMs), where fold change $\geq 2$ or fold change $\leq 0.5$ and the metabolites with VIP $\geq 1$. PCA and PLS-DA were implemented in R software (www.rproject.org/) to investigate tea metabolite variety-specific accumulation [55].

\subsection{RNA-Seq and Data Processing}

RNA isolation and purification from JMZ and HD, as well as cDNA library construction and sequencing, were performed as previously described [56]. Total RNA was extracted from the young shoots of JMZ and HD by using the pBIzol kit (BIOFLUX, Hangzhou Bori Technology, Hangzhou, 
China). RNA integrity of samples was measured using a Nano-Drop ultraviolet spectrophotometer (Thermo, Waltham, MA, USA) and a Bioanalyzer 2100 System (Agilent, Santa Clara, CA, USA). About $3 \mu \mathrm{g}$ of RNA per sample was prepared, and sequencing was carried out on an Illumina HiSeq 4000 platform to generate $150 \mathrm{bp}$ paired end reads for each sample. Two test samples were used to construct the transcriptome library and Illumina sequencing in Allwegene Biotechnology Co., Ltd. (Beijing, China).

\subsection{Analysis of DEGs}

Raw reads in FASTQ format were first processed by filtering out adapters and low-quality sequences. Clean reads were obtained by removing reads containing adapter and poly- $\mathrm{N}$ and low-quality reads from raw data. All clean reads were aligned to the reference transcripts of $C$. sinensis (http://tpia.teaplant.org/index.html) by applying TopHat2. Gene expression levels were calculated based on the number of reads mapped to the reference sequence to receive the unigenes by using HISAT2 and StringTie software. To assess gene expression levels, we calculated the FPKM of each gene. DEGs that were related to the metabolism of flavonoids (e.g., flavones, anthocyanidins, and chalcones) were functionally annotated by using GO and KEGG [57]. DEGs between JMZ and HD were enrolled based on threshold values of $\mid \log _{2}$ (fold change) $\mid \geq 1$ and adjusted $p \leq 0.005$.

\section{5. qRT-PCR Validation of RNA-Seq Results}

In total, 17 genes were selected for qRT-PCR analysis to examine the different expression profiles. qRT-PCR detection system of the samples was performed as previously described [58]. The relative expression of each gene was calculated after normalization to that of the CsGAPDH (GE651107) gene. The samples of the three independent biological replicates were used for the analyses. The expression levels of candidate genes were determined by applying the $2^{-\Delta \Delta C T}$ method [59]. The specific primers used for RT-qPCR are listed in Table S1.

\subsection{UPLC-TOF-MS for Determination of Catechins Content}

To further supplement the conclusion of metabolomics analysis, the content of catechins was analyzed by UPLC-TOF-MS. The analysis method adopts the method of Yue et al. [60], and we have made some modifications. Briefly, $0.05 \mathrm{~g}$ tea powder was weighed, placed into a $5 \mathrm{~mL}$ glass tube, and added to $5 \mathrm{~mL}$ of extraction solution (methanol: water $=7: 3$ ). The solution was subjected to ultrasonic extraction for $30 \mathrm{~min}$ (ds-8510 DTH, Shanghai, China), centrifuged for 3 min (Avanti-e, Beckman Coulter, USA), and tested on the machine after passing through a $0.22 \mu \mathrm{m}$ PTFE filter membrane (f2513-4, Thermo Scientific, USA). The UPLC TSS T3 ultrahigh performance liquid chromatography column $\left(150 \mathrm{~mm} \times 2.1 \mathrm{~mm}, 18 \mu \mathrm{m}\right.$, Waters, the UK) was utilized with column temperature of $40^{\circ} \mathrm{C}$, injection amount of $5 \mu \mathrm{L}$, flow rate of $0.3 \mathrm{~mL} / \mathrm{min}$, mobile phase A comprising $0.1 \%$ formic acid water, and mobile phase $\mathrm{B}$ comprising acetonitrile, and catechin was identified by the following standards: ESI ionization mode, capillary voltage of $2.5 \mathrm{KV}$, sampling cone voltage of $50 \mathrm{~V}$, ion source temperature of $120^{\circ} \mathrm{C}$, dissolvent gas temperature of $40^{\circ} \mathrm{C}$, cone gas of $50 \mathrm{~L} / \mathrm{h}$, dissolvent gas flow rate of $800 \mathrm{~L} / \mathrm{h}$, MS adopted the MSe scanning mode, mass spectrum, scanning range of 100-1000 m/z, scanning rate of $0.1 \mathrm{~s}$, centroid data format, low collision energy of $5 \mathrm{~V}$, and high collision energy of 20-40 V.

\section{Conclusions}

The formation mechanisms of leaf coloration in purple-leaf tea were investigated using widely targeted metabolomic and transcriptomic approaches. The accumulation of flavonoid metabolites, specifically, the high accumulation of anthocyanin and catechin compounds in purple-leaf tea, was observed. In addition, the related forms of the regulation of the structural genes involved in the flavonoid biosynthesis pathway were investigated. The involvement of these structural genes or their regulators (i.e., $\mathrm{TFs}$ ) may provide a mechanism to improve the accumulation of red anthocyanins in purple-leaf tea, thereby maintaining beneficial metabolites during the purple-leaf period. 
Supplementary Materials: The following are available online at http://www.mdpi.com/1422-0067/21/11/4167/s1, Table S1: the primers for qRT-PCR; Table S2: significantly changed metabolites in JMZ compared with HD; and Table S3: number of genes with different expression levels.

Author Contributions: N.Y., P.W., and X.C. conceived and designed the research. M.G., X.L., and S.W. performed the experiments. Y.Z., S.J., and X.C. analyzed the date. X.C. wrote the manuscript and S.J. revised the manuscript. All authors reviewed and approved the manuscript.

Funding: This research was funded by the Fujian Province "2011 Collaborative Innovation Center," Chinese Oolong Tea Industry Innovation Center special project (J2015-75), Fujian Agriculture and Forestry University Project for Technological Innovation of Tea Industry Chain and Service System Construction, and Fujian Agriculture and Forestry University Science and Technology Innovation Special Fund (CXZX2017181).

Conflicts of Interest: The authors declare no conflict of interest

\section{Abbreviations}

$\begin{array}{ll}\text { PAL } & \text { phenylalanine ammonia-lyase } \\ \text { LAR } & \text { leucoanthocyanidin reductase } \\ \text { UFGT } & \text { UDP glucose-flavonoid 3-o-glcosyl-transferase } \\ \text { C4H } & \text { cinnamic acid hydroxylase } \\ \text { 4CL } & \text { coumadin CoA ligase } \\ \text { CHS } & \text { chalcone synthase } \\ \text { CHI } & \text { chalcone isomerase } \\ \text { F3H } & \text { flavonoid 3-hydroxylase } \\ \text { F3'H } & \text { flavonoid 3'-hydroxylase } \\ \text { F3'5'H } & \text { flavonoid 3' 5'-hydroxylase } \\ \text { DRF } & \text { dihydroflavonol 4-reductase } \\ \text { ANR } & \text { anthocyanidin reductase } \\ \text { GO } & \text { gene ontology } \\ \text { KEGG } & \text { enrichment by using KOBAS software } \\ \text { FPKM } & \text { fragments per kilobase per million } \\ \text { TF } & \text { transcription factor }\end{array}$

\section{References}

1. Kumari, M.; Thakur, S.; Kumar, A.; Joshi, R.; Kumar, P.; Shankar, R.; Kumar, R. Regulation of color transition in purple tea (Camellia sinensis). Planta 2019, 251, 35. [CrossRef] [PubMed]

2. Zhao, M.Y.; Zhang, N.; Gao, T.; Jin, J.Y.; Jing, T.T.; Wang, J.M.; Wu, Y.; Wan, X.C.; Schwab, W.; Song, C. Sesquiterpene glucosylation mediated by glucosyltransferase UGT91Q2 is involved in the modulation of cold stress tolerance in tea plants. New Phytol. 2019, 226, 362-372. [CrossRef] [PubMed]

3. He, X.J.; Zhao, X.C.; Gao, L.P.; Shi, X.X.; Dai, X.L.; Liu, Y.J.; Xia, T.; Wang, Y.S. Isolation and characterization of key genes that promote flavonoid accumulation in purple-leaf tea (Camellia sinensis L.). Sci. Rep. 2018, 8 , 130. [CrossRef] [PubMed]

4. Shen, J.Z.; Zou, Z.W.; Zhang, X.Z.; Zhou, L.; Wang, Y.H.; Fang, W.P.; Zhu, X.J. Metabolic analyses reveal different mechanisms of leaf color change in two purple-leaf tea plant (Camellia sinensis L.) cultivars. Hortic. Res. 2018, 5, 7. [CrossRef] [PubMed]

5. Li, J.; Lv, X.J.; Wang, L.X.; Qiu, Z.M.; Song, X.M.; Lin, J.K.; Chen, W. Transcriptome analysis reveals the accumulation mechanism of anthocyanins in 'Zijuan' tea (Camellia sinensis var. asssamica (Masters) kitamura) leaves. Plant Growth Regul. 2017, 81, 51-61. [CrossRef]

6. Kerio, L.C.; Wachira, F.N.; Wanyoko, J.K.; Rotich, M.K. Total polyphenols, catechin profiles and antioxidant activity of tea products from purple leaf coloured tea cultivars. Food Chem. 2013, 136, 1405-1413. [CrossRef] [PubMed]

7. Wang, Q.P.; Peng, C.X.; Gao, B.; Gong, J.S. Influence of large molecular polymeric pigments isolated from fermented Zijuan tea on the activity of key enzymes involved in lipid metabolism in rat. Exp. Gerontol. 2012, 47, 672-679. [CrossRef] [PubMed] 
8. Fang, R.; Redfern, S.P.; Kirkup, D.; Porter, E.A.; Kite, G.C.; Terry, L.A.; Berry, M.J.; Simmonds, M.S. Variation of theanine, phenolic, and methylxanthine compounds in 21 cultivars of Camellia sinensis harvested in different seasons. Food Chem. 2017, 220, 517-526. [CrossRef] [PubMed]

9. Gao, G.; Chen, P.; Chen, J.; Chen, K.M.; Wang, X.F.; Abubakar, A.S.; Liu, N.; Yu, C.M.; Zhu, A.G. Genomic survey, transcriptome, and metabolome analysis of Apocynum venetum and Apocynum hendersonii to reveal major flavonoid biosynthesis pathways. Metabolites 2019, 9, 296. [CrossRef] [PubMed]

10. Winkel-Shirley, B. Flavonoid biosynthesis. A colorful model for genetics, biochemistry, cell biology, and biotechnology. Plant Physiol. 2001, 126, 485-493. [CrossRef] [PubMed]

11. Scharbert, S.; Hofmann, T. Molecular definition of black tea taste by means of quantitative studies, taste reconstitution, and omission experiments. J. Agric. Food Chem. 2005, 53, 5377-5384. [CrossRef] [PubMed]

12. Patra, B.; Schluttenhofer, C.; Wu, Y.M.; Pattanaik, S.; Yuan, L. Transcriptional regulation of secondary metabolite biosynthesis in plants. Biochim. Biophys. Acta. 2013, 1829, 1236-1247. [CrossRef] [PubMed]

13. Kim, S.; Hwang, G.; Lee, S.; Zhu, J.Y.; Paik, I.; Nguyen, T.T.; Kim, J.M.; Oh, E. High ambient temperature represses anthocyanin biosynthesis through degradation of HY5. Front Plant Sci. 2017, 8, 1787. [CrossRef] [PubMed]

14. Jin, M.; Zhang, X.H.; Zhao, M.C.; Deng, M.; Du, Y.H.; Zhou, Y.; Wang, S.C.; Tohge, T.; Fernie, A.R.; Willmitzer, L.; et al. Integrated genomics-based mapping reveals the genetics underlying maize flavonoid biosynthesis. BMC Plant Biol. 2017, 17, 17. [CrossRef] [PubMed]

15. Wang, Z.R.; Cui, Y.Y.; Vainstein, A.; Chen, S.W.; Ma, H.Q. Regulation of fig (Ficus carica L.) fruit color: Metabolomic and transcriptomic analyses of the flavonoid biosynthetic pathway. Front Plant Sci. 2017, 8, 1990. [CrossRef] [PubMed]

16. Wu, L.Y.; Fang, Z.T.; Lin, J.K.; Sun, Y.; Du, Z.Z.; Guo, Y.L.; Liu, J.H.; Liang, Y.R.; Ye, J.H. Complementary iTRAQ proteomic and transcriptomic analyses of leaves in tea plant (Camellia sinensis L.) with different maturity and regulatory network of flavonoid biosynthesis. J. Proteome Res. 2019, 18, 252-264. [PubMed]

17. Long, L.; Liu, J.; Gao, Y.; Xu, F.C.; Zhao, J.R.; Li, B.; Gao, W. Flavonoid accumulation in spontaneous cotton mutant results in red coloration and enhanced disease resistance. Plant Physiol. Biochem. 2019, 143, 40-49. [CrossRef] [PubMed]

18. Wang, Y.S.; Xu, Y.J.; Gao, L.P.; Yu, O.; Wang, X.Z.; He, X.J.; Jiang, X.L.; Liu, Y.J.; Xia, T. Functional analysis of flavonoid $3^{\prime}, 5^{\prime}$-hydroxylase from tea plant (Camellia sinensis): Critical role in the accumulation of catechins. BMC Plant Biol. 2014, 14, 347. [CrossRef] [PubMed]

19. Zhu, J.Y.; Xu, Q.S.; Zhao, S.Q.; Xia, X.B.; Yan, X.M.; An, Y.L.; Mi, X.Z.; Guo, L.X.; Samarina, L.; Wei, C.L. Comprehensive co-expression analysis provides novel insights into temporal variation of flavonoids in fresh leaves of the tea plant (Camellia sinensis). Plant Sci. 2020, 290, 110306. [CrossRef] [PubMed]

20. Wang, P.J.; Zheng, Y.C.; Guo, Y.C.; Liu, B.S.; Jin, S.; Liu, S.Z.; Zhao, F.; Chen, X.J.; Sun, Y.; Yang, J.F.; et al. Widely targeted metabolomic and transcriptomic analyses of novel albino tea mutant of "Rougui". Forests 2020, 11, 229. [CrossRef]

21. Pan, Y.Y.; Wu, H.L.; Li, J.X.; Yang, C.W.; Liu, J. Advances in research and utilization of purple tea. Guangdong Agric. Sci. 2015, 42, 8-12; 17.

22. Qi, T.C.; Song, S.S.; Ren, Q.C.; Wu, D.W.; Huang, H.; Chen, Y.; Fang, M.; Peng, W.; Ren, C.M.; Xie, D.X. The Jasmonate-ZIM-domain proteins interact with the WD-Repeat/bHLH/MYB complexes to regulate Jasmonate-mediated anthocyanin accumulation and trichome initiation in Arabidopsis thaliana. Plant Cell 2011, 23, 1795-1814. [CrossRef] [PubMed]

23. Stracke, R.; Ishihara, H.; Huep, G.; Barsch, A.; Mehrtens, F.; Niehaus, K.; Weisshaar, B. Differential regulation of closely related R2R3-MYB transcription factors controls flavonol accumulation in different parts of the Arabidopsis thaliana seedling. Plant J. 2007, 50, 660-677. [CrossRef] [PubMed]

24. Wang, W.L.; Wang, Y.X.; Li, H.; Liu, Z.W.; Cui, X.; Zhuang, J. Two MYB transcription factors (CsMYB2 and CsMYB26) are involved in flavonoid biosynthesis in tea plant [Camellia sinensis (L.) O. Kuntze]. BMC Plant Biol. 2018, 18, 288. [CrossRef] [PubMed]

25. Robbins, R.J. Phenolic acids in foods: An overview of analytical methodology. J. Agric. Food Chem. 2003, 51, 2866-2887. [CrossRef] [PubMed]

26. Heleno, S.A.; Martins, A.; Queiroz, M.J.; Ferreira, I.C. Bioactivity of phenolic acids: Metabolites versus parent compounds: A review. Food Chem. 2015, 173, 501-513. [CrossRef] [PubMed] 
27. Schmidt, C.G.; Goncalves, L.M.; Prietto, L.; Hackbart, H.S.; Furlong, E.B. Antioxidant activity and enzyme inhibition of phenolic acids from fermented rice bran with fungus Rizhopus oryzae. Food Chem. 2014, 146, 371-377. [CrossRef] [PubMed]

28. Sroka, Z.; Cisowski, W. Hydrogen peroxide scavenging, antioxidant and anti-radical activity of some phenolic acids. Food Chem. Toxicol. 2003, 41, 753-758. [CrossRef]

29. You, Y.L.; Li, N.; Han, X.; Guo, J.L.; Zhao, Y.; Huang, W.D.; Zhan, J.C. The effects of six phenolic acids and tannic acid on colour stability and the anthocyanin content of mulberry juice during refrigerated storage. Int. J. Food Sci. Technol. 2019, 54, 2141-2150. [CrossRef]

30. Wei, C.L.; Yang, H.; Wang, S.B.; Zhao, J.; Liu, C.; Gao, L.P.; Xia, E.H.; Lu, Y.; Tai, Y.L.; She, G.B.; et al. Draft genome sequence of Camellia sinensis var. sinensis provides insights into the evolution of the tea genome and tea quality. Proc. Natl. Acad. Sci. USA 2018, 115, E4151-E4158. [CrossRef] [PubMed]

31. Qiao, Z.Q.; Liu, S.S.; Zeng, H.J.; Li, Y.X.; Wang, X.Y.; Chen, Y.; Wang, X.M.; Cai, N. Exploring the molecular mechanism underlying the stable purple-red leaf phenotype in Lagerstroemia indica cv. ebony embers. Int. J. Mol. Sci. 2019, 20, 5636. [CrossRef] [PubMed]

32. Liu, S.R.; An, Y.L.; Tong, W.; Qin, X.J.; Samarina, L.D.; Guo, R.; Xia, X.B.; Wei, C.L. Characterization of genome-wide genetic variations between two varieties of tea plant (Camellia sinensis) and development of InDel markers for genetic research. BMC Genom. 2019, 20, 1-16. [CrossRef] [PubMed]

33. Li, C.F.; Zhu, Y.; Yu, Y.; Zhao, Q.Y.; Wang, S.J.; Wang, X.C.; Yao, M.Z.; Luo, D.; Li, X.; Chen, L. Global transcriptome and gene regulation network for secondary metabolite biosynthesis of tea plant (Camellia sinensis). BMC Genom. 2015, 16, 560. [CrossRef] [PubMed]

34. Zhou, C.; Mei, X.; Rothenberg, D.O.; Yang, Z.B.; Zhang, W.T.; Wan, S.H.; Yang, H.J.; Zhang, L.Y. Metabolome and Transcriptome Analysis Reveals Putative Genes Involved in Anthocyanin Accumulation and Coloration in White and Pink Tea (Camellia sinensis) Flower. Molecules 2020, 25, 190. [CrossRef] [PubMed]

35. Saito, T.; Honma, D.; Tagashira, M.; Kanda, T.; Nesumi, A.; Maeda-Yamamoto, M. Anthocyanins from new red leaf tea 'Sunrouge'. J. Agric. Food Chem. 2011, 59, 4779-4782. [CrossRef] [PubMed]

36. Wang, W.Z.; Zhou, Y.H.; Wu, Y.L.; Dai, X.L.; Liu, Y.J.; Qian, Y.M.; Li, M.Z.; Jiang, X.L.; Wang, Y.S.; Gao, L.P.; et al. Insight into catechins metabolic pathways of Camellia sinensis based on genome and transcriptome analysis. J. Agric. Food Chem. 2018, 66, 4281-4293. [CrossRef] [PubMed]

37. Song, L.B.; Ma, Q.Q.; Zou, Z.W.; Sun, K.; Yao, Y.T.; Tao, J.H.; Kaleri, N.A.; Li, X.H. Molecular Link between Leaf Coloration and Gene Expression of Flavonoid and Carotenoid Biosynthesis in Camellia sinensis Cultivar 'Huangjinya'. Front. Plant Sci. 2017, 8, 803. [CrossRef] [PubMed]

38. Wang, L.X.; Pan, D.Z.; Liang, M.; Abubakar, Y.S.; Li, J.; Lin, J.K.; Chen, S.P.; Chen, W. Regulation of Anthocyanin Biosynthesis in Purple Leaves of Zijuan Tea (Camellia sinensis var. kitamura). Int. J. Mol. Sci. 2017, 18, 833. [CrossRef] [PubMed]

39. Liu, Y.H.; Lv, J.L.; Liu, Z.B.; Wang, J.; Yang, B.Z.; Chen, W.C.; Ou, L.J.; Dai, X.Z.; Zhang, Z.Q.; Zou, X.X. Integrative analysis of metabolome and transcriptome reveals the mechanism of color formation in pepper fruit (Capsicum annuum L.). Food Chem. 2020, 306, 125629. [CrossRef] [PubMed]

40. Guo, Y.Q.; Chang, X.J.; Zhu, C.; Zhang, S.T.; Li, X.Z.; Fu, H.F.; Chen, C.S.; Lin, Y.L.; Lai, Z.X. De novo transcriptome combined with spectrophotometry and gas chromatography-mass spectrometer (GC-MS) reveals differentially expressed genes during accumulation of secondary metabolites in purple-leaf tea (Camellia sinensis cv Hongyafoshou). J. Hortic. Sci. Biotechnol. 2019, 94, 349-367. [CrossRef]

41. He, F.; Mu, L.; Yan, G.L.; Liang, N.N.; Pan, Q.H.; Wang, J.; Reeves, M.J.; Duan, C.D. Biosynthesis of anthocyanins and their regulation in colored grapes. Molecules 2010, 15, 9057-9091. [CrossRef] [PubMed]

42. Boss, P.K.; Davies, C.; Robinson, S.P. Analysis of the expression of anthocyanin pathway genes in developing Vitis vinifera L. cv. shiraz grape berries and the implications for pathway regulation. Plant Physiol. 1996, 111, 1059-1066. [CrossRef] [PubMed]

43. Yonekura-Sakakibara, K.; Tohge, T.; Matsuda, F.; Nakabayashi, R.; Takayama, H.; Niida, R.; Watanabe-Takahashi, A.; Inoue, E.; Saito, K. Comprehensive flavonol profiling and transcriptome coexpression analysis leading to decoding gene-metabolite correlations in Arabidopsis. Plant Cell 2008, 20, 2160-2176. [CrossRef] [PubMed]

44. Besseau, S.; Hoffmann, L.; Geoffroy, P.; Lapierre, C.; Pollet, B.; Legrand, M. Flavonoid accumulation in Arabidopsis repressed in lignin synthesis affects auxin transport and plant growth. Plant Cell 2007, 19, 148-162. [CrossRef] [PubMed] 
45. Hoffmann, L.; Besseau, S.; Geoffroy, P.; Ritzenthaler, C.; Meyer, D.; Lapierre, C.; Pollet, B.; Legrand, M. Silencing of hydroxycinnamoyl-coenzyme A shikimate/quinate hydroxycinnamoyl transferase affects phenylpropanoid biosynthesis. Plant Cell 2004, 16, 1446-1465. [CrossRef] [PubMed]

46. Liu, J.Y.; Osbourn, A.; Ma, P.D. MYB Transcription factors as regulators of phenylpropanoid metabolism in plants. Mol. Plant 2015, 8, 689-708. [CrossRef] [PubMed]

47. Li, H.Y.; Lv, Q.Y.; Ma, C.; Qu, J.T.; Cai, F.; Deng, J.; Huang, J.; Ran, P.; Shi, T.X.; Chen, Q.F. Metabolite profiling and transcriptome analyses provide insights into the flavonoid biosynthesis in the developing seed of tartary buckwheat (Fagopyrum tataricum). J. Agric. Food Chem. 2019, 67, 11262-11276. [CrossRef] [PubMed]

48. Wang, N.; Qu, C.Z.; Jiang, S.H.; Chen, Z.J.; Xu, H.F.; Fang, H.C.; Su, M.Y.; Zhang, J.; Wang, Y.C.; Liu, W.J.; et al. The proanthocyanidin-specific transcription factor MdMYBPA1 initiates anthocyanin synthesis under low-temperature conditions in red-fleshed apples. Plant J. 2018, 96, 39-55. [CrossRef] [PubMed]

49. Zhang, J.; Liu, Y.Y.; Bu, Y.F.; Zhang, X.; Yao, Y.C. Factor analysis of MYB gene expression and flavonoid affecting petal color in three crabapple cultivars. Front. Plant Sci. 2017, 8, 137. [CrossRef] [PubMed]

50. Zhao, L.; Gao, L.P.; Wang, H.X.; Chen, X.T.; Wang, Y.S.; Yang, H.; Wei, C.L.; Wan, X.C.; Xia, T. The R2R3-MYB, bHLH, WD40, and related transcription factors in flavonoid biosynthesis. Funct. Integr. Genom. 2013, 13, 75-98. [CrossRef] [PubMed]

51. Xu, W.; Dubos, C.; Lepiniec, L. Transcriptional control of flavonoid biosynthesis by MYB-bHLH-WDR complexes. Trends Plant Sci. 2015, 20, 176-185. [CrossRef] [PubMed]

52. Shin, J.; Park, E.; Choi, G. PIF3 regulates anthocyanin biosynthesis in an HY5-dependent manner with both factors directly binding anthocyanin biosynthetic gene promoters in Arabidopsis. Plant J. 2007, 49, 981-994. [CrossRef] [PubMed]

53. Chen, W.; Gong, L.; Guo, Z.L.; Wang, W.S.; Zhang, H.Y.; Liu, X.Q.; Yu, S.B.; Xiang, L.Z.; Luo, J. A novel integrated method for large-scale detection, identification, and quantification of widely targeted metabolites: Application in the study of rice metabolomics. Mol. Plant 2013, 6, 1769-1780. [CrossRef] [PubMed]

54. Zhu, G.T.; Wang, S.C.; Huang, Z.J.; Zhang, S.; Liao, Q.G.; Zhang, C.Z.; Lin, T.; Qin, M.; Peng, M.; Yang, C.K.; et al. Rewiring of the fruit metabolome in tomato breeding. Cell 2018, 172, 249-261.e12. [CrossRef] [PubMed]

55. Wang, S.C.; Tu, H.; Wan, J.; Chen, W.; Liu, X.Q.; Luo, J.; Xu, J.; Zhang, H.Y. Spatio-temporal distribution and natural variation of metabolites in citrus fruits. Food Chem. 2016, 199, 8-17. [CrossRef] [PubMed]

56. Zheng, Y.C.; Wang, P.J.; Chen, X.J.; Sun, Y.; Yue, C.; Ye, N. Transcriptome and metabolite profiling reveal novel insights into volatile heterosis in the tea plant (Camellia Sinensis). Molecules 2019, 24, 3380. [CrossRef] [PubMed]

57. Luo, P.; Ning, G.G.; Wang, Z.; Shen, Y.X.; Jin, H.R.; Li, P.H.; Huang, S.S.; Zhao, J.; Bao, M.Z. Disequilibrium of flavonol synthase and dihydroflavonol-4-reductase expression associated tightly to white vs. red color flower formation in plants. Front. Plant Sci. 2015, 6, 1257. [CrossRef] [PubMed]

58. Wang, P.J.; Chen, D.; Zheng, Y.C.; Jin, S.; Yang, J.F.; Ye, N.X. Identification and expression analyses of SBP-Box genes reveal their involvement in abiotic Stress and hormone response in tea plant (Camellia sinensis). Int. J. Mol. Sci. 2018, 19, 3404. [CrossRef] [PubMed]

59. Livak, K.J.; Schmittgen, T.D. Analysis of relative gene expression data using real-time quantitative PCR and the 2- $\triangle \triangle \mathrm{CT}$ method. Methods 2001, 25, 402-408. [CrossRef] [PubMed]

60. Yue, W.J.; Sun, W.J.; Rao, R.S.P.; Ye, N.X.; Yang, Z.B.; Chen, M.J. Non-targeted metabolomics reveals distinct chemical compositions among different grades of Bai Mudan white tea. Food Chem. 2019, 277, $289-297$. [CrossRef] [PubMed]

(C) 2020 by the authors. Licensee MDPI, Basel, Switzerland. This article is an open access article distributed under the terms and conditions of the Creative Commons Attribution (CC BY) license (http://creativecommons.org/licenses/by/4.0/). 